Elsevier Editorial System(tm) for Materials Science \& Engineering A Manuscript Draft

Manuscript Number: MSEA-D-08-01241R1

Title: Load partitioning during creep of powder metallurgy metal matrix composites and shear-lag model predictions

Article Type: Research Paper

Keywords: Metal Matrix Composites (MMCs); Aluminum Alloys; Creep; Powder Processing

Corresponding Author: Dr. Gaspar Gonzalez-Doncel, PhD

Corresponding Author's Institution: CENIM, C.S.I.C.

First Author: Ricardo Fernández, PhD

Order of Authors: Ricardo Fernández, PhD; Gaspar Gonzalez-Doncel, PhD

Abstract: It was shown in a previous work that the load transfer mechanism plays a relevant role during the high temperature deformation of discontinuously reinforced metal matrix composites, MMCs. This idea emerged from the comparison of the creep data of a powder metallurgy, PM, 6061Al-15vol\%SiCw composite and the corresponding un-reinforced 6061Al alloy. The idea was further supported by a qualitative analysis of the creep data of MMCs from a number of investigations reported in the literature, particularly of PM composites. In the present work a quantitative and more thorough study of the creep data of these PM composites is presented. Specifically, a well known Shear-Lag model is used to compare the composites creep strength increment and the predicted load transferred to the reinforcement. These new results sustain more thoroughly the relevance of the load transfer mechanism during creep of MMCs. 


\title{
Load partitioning during creep of powder metallurgy metal matrix composites and shear-lag model predictions
}

\author{
Ricardo Fernández ${ }^{\S}$, Gaspar González-Doncel* \\ Dept. of Physical Metallurgy, Centro Nacional de Investigaciones Metalúrgicas (CENIM), C.S.I.C., \\ Av. de Gregorio del Amo 8, E-28040 Madrid, Spain \\ ${ }^{\S}$ Present address, Thin Film R\&D Dept. INDO, SA, C/ Alcalde Barnils 72, 08174 Sant Cugat del \\ Vallés, Barcelona, Spain
}

\begin{abstract}
It was shown in a previous work that the load transfer mechanism plays a relevant role during the high temperature deformation of discontinuously reinforced metal matrix composites, MMCs. This idea emerged from the comparison of the creep data of a powder metallurgy, PM, 6061Al$15 \mathrm{vol} \% \mathrm{SiC}_{\mathrm{w}}$ composite and the corresponding un-reinforced $6061 \mathrm{Al}$ alloy. The idea was further supported by a qualitative analysis of the creep data of MMCs from a number of investigations reported in the literature, particularly of PM composites. In the present work a quantitative and more thorough study of the creep data of these PM composites is presented. Specifically, a well known Shear-Lag model is used to compare the composites creep strength increment and the predicted load transferred to the reinforcement. These new results sustain more thoroughly the relevance of the load transfer mechanism during creep of MMCs.
\end{abstract}

Keywords: Metal Matrix Composites (MMCs); Aluminum Alloys; Creep; Powder Processing

* Corresponding author, ggd@ cenim.csic.es. Phone, +34 915538900 ext.337 


\section{1.- Introduction}

In a previous work [1], the creep behavior of a powder metallurgy, PM, 6061 Al-15vol\% $\mathrm{SiC}_{\mathrm{w}}$ composite was investigated. The comparison of the creep data of this composite and that of the corresponding un-reinforced PM 6061 Al alloy allowed explaining the improved composite creep strength without the need of a threshold stress term. Instead, a load transfer mechanism, a well know phenomenon resulting from the different stiffness of ceramic reinforcement and metallic matrix, was proposed to be relevant for the improved composite creep strengthening. The threshold stress term is being very often invoked to explain this improved behavior [2-4] but the microstructural bases to explain it are not understood. This alternative procedure, however, opens a clearer and simpler way to understand the creep behavior of discontinuously reinforced MMCs without bringing into play terms which are incomprehensible from microstructural basis.

The idea of the relevance of the load transfer mechanisms was raised by the fact that a proportionality between the applied stress, $\sigma$, and the additional stress needed by the composite with respect the un-reinforced alloy to deform at a given strain rate, $\Delta \sigma(\sigma)$, was found. Furthermore, such proportionality was also detected for a wide variety of composite materials reviewed from literature [1]. The $\Delta \sigma(\sigma)$ dependence found for the composite material investigated in [1] correlates reasonably well with Shear-Lag and Eshelby model predictions of the load transferred during composite deformation.

The detailed analysis of the linear dependence $\Delta \sigma(\sigma)$ detected for the composites reviewed and the comparison of experimental data with models' predictions, however, was not conducted in [1]. This is, hence, the purpose of the present investigation: To compare, quantitatively, the experimental data of $\Delta \sigma(\sigma)$ obtained for these composites with a Shear-Lag model prediction of the load transferred to the reinforcement. Materials from the literature have been selected whenever creep information of the corresponding un-reinforced alloys was also available. Such direct comparison is crucial to know the experimental composites creep strength increment.

\section{2.- Materials investigated.}

The materials selected for this study have been those processed by the PM route [1,5-15]. These composite materials have a "firm" bonding between matrix and reinforcement and are less prone to develop unpredictable damage mechanisms or de-cohesion phenomena during creep deformation than materials obtained by the ingot metallurgy, IM, process. As argued in [1], the PM route avoids deleterious reactions during processing between the metal phase and the ceramic reinforcement and, hence, a "cleaner" metal-ceramic interface is developed. On the contrary, chemical reactions between the liquid metal and the particles are likely to occur in IM composites. The derived 
products at the interface, such as the formation of aluminum carbide, $\mathrm{Al}_{4} \mathrm{C}_{3}$, particles when $\mathrm{SiC}$ is are used as the reinforcing phase, are usually harmful for the good bonding, favoring premature composite failure [16].

It is clear, then, that the PM composites are materials for which damage and/or de-cohesion phenomena are minimized, and the load transfer mechanism can be evaluated more rigorously and in better detail. Following the same procedure as in [1], materials selected for this analysis include aluminum alloy matrix composites selected from the literature whenever creep data of the corresponding un-reinforced alloys (under similar testing condition as for the composites) are also available. The materials selected are those summarized in Table I.

As mentioned, the difference obtained between composites creep strength increment has been attributed mainly to a load partitioning phenomenon, but a matrix strengthening factor should be also taken into account [1]. This contribution is due to the finer microstructure developed in the composite than in the un-reinforced alloy: i.e., smaller inter-obstacle distance, $\lambda$, for dislocation motion. So, considering Sherby's sub-structure invariant creep model, the steady or minimum creep rate, $\mathcal{E}$, is given by,

$$
\dot{\varepsilon}=K\left(\frac{D_{L}}{\boldsymbol{b}^{2}}\right)\left(\frac{\lambda}{\boldsymbol{b}}\right)^{3}\left(\frac{\sigma^{\prime}}{E}\right)^{8}
$$

where $K$ is a material constant (equal to about $10^{9}$ for high staking fault energy materials), $D_{L}=D_{o}$ $\exp \left(-Q_{L} / R T\right)$ is the lattice diffusion coefficient of aluminum, $\left(D_{o}=1.7 \times 10^{-4} \mathrm{~m}^{2} / \mathrm{s}, Q_{L}=142 \mathrm{~kJ} / \mathrm{mol}\right.$ [17]), $\boldsymbol{b}$ is de Burgers vector, equal to: $\boldsymbol{b}=2.86 \times 10^{-10} \mathrm{~m}$ in aluminum, $E$ is the Young's modulus, and $\sigma^{\prime}$ is the effective stress resulting from the presence of a "true" threshold stress associated with the $\mathrm{Al}_{2} \mathrm{O}_{3}$ particles of PM Al alloys [18]. This contribution will be discussed in more detail within the context of all other involved mechanisms in a separate publication. Consequently, the creep rate of the composite matrix, $\dot{\varepsilon}_{\text {comp }}$ in terms of that of the un-reinforced alloy, $\dot{\varepsilon}_{\text {alloy }}$, should be given, following equation (1), by,

$$
\dot{\mathcal{E}}_{\text {comp }}=\frac{1}{a^{3}} \dot{\varepsilon}_{\text {alloy }}
$$

where $a=\lambda_{\text {alloy }} / \lambda_{\text {comp }}$. In [1] the ratio $\lambda_{\text {alloy }} / \lambda_{\text {comp }}$ was estimated considering that $\lambda$ is inversely proportional to $\sqrt{\rho}$, where $\rho$ is the dislocation density [19]. Thus, it was obtained, $a=\lambda_{\text {alloy }} / \lambda_{\text {comp }}=\sqrt{\rho_{\text {comp }} / \rho_{\text {alloy }}}=1.97$ according to the $\rho$ values provided for the materials 
investigated (see Table I of [1]). Considering the lack of data to estimate rigorously this contribution in the materials of Table I, it has been assumed here a similar matrix strengthening factor as in [1]. This contribution is, however, significantly less relevant than that due to the load partitioning phenomenon.

\section{3.- Load partitioning: Ryu's et al Shear-Lag model}

Models based on two different approaches, namely, the Shear-Lag [20] and the Eshelby [21] methods, are commonly used for studying load partitioning in MMCs. These two approaches differ in the mathematical treatment, scalar in the first case and tensorial in the second one. The election of one or the other depends on the microstructural features of the reinforcement. Eshelby models are supposed to provide more rigorous predictions in composites with particles of low aspect ratio or when particles shape is close to an ellipsoid. On the contrary, Shear-Lag models are expected to give good predictions in composites with elongated or large aspect ratio particles. Furthermore, they are much easier to operate than Eshelby models due to their scalar nature. In this scenario, the choice of one kind of model or another depends on author's criterion. Due to the number of materials investigated, with very different microstructures, and based on the reliable previous good predictions of the load transferred obtained with models of both kinds [1], and also for its simplicity, the creep strengthening of these PM composites materials, Table I, (after microstructural strengthening subtraction) has been compared with the prediction supplied by Ryu's et al Shear-lag model [22], in the same manner as done in [1]. This model is explained in detail in [22]. A brief description was done in [1] and will be also given here.

Accordingly, the magnitude of the load transferred to the reinforcement is quantified by knowing the value of two microstructural parameters: the volume fraction of particles and their aspect ratio. The effective value of the aspect ratio can be influenced by particles' orientation in the case of elongated particles. These parameters are easily implemented in Ryu's et al. model [22]. This model, based on the one proposed by Nardone and Prewo [23], considers the reinforcement as perfect cylinders of aspect ratio $S$, and takes into account the possible misalignment with the loading direction. In this case, the effective aspect ratio of individual whiskers, $S_{\text {eff }}^{I}$, misaligned $\theta$ with the loading direction (assuming an axial symmetry, as that resulting form extrusion) is given by,

$$
S_{\text {eff }}^{I}=S \cos ^{2} \theta+\left(\frac{3 \pi-4}{3 \pi}\right)\left(1+\frac{1}{S}\right) \sin ^{2} \theta
$$

The average effective aspect ratio of the reinforcement, $S_{\text {eff }}$, results, then, from,

$$
S_{e f f}=\int_{0}^{\pi / 2} S_{e f f}^{I}(\theta) \gamma(\theta) d \theta=\int_{0}^{\pi / 2} S_{e f f}^{I} I(\theta) 2 \pi \sin \theta d \theta
$$


where $\chi(\theta)$ is the density function which defines the degree of alignment of the reinforcement with the loading direction. Equation (4) can be solved numerically by the Simpson method [24].

Then, the effective stress on the matrix, $\sigma_{e f f}$, can be calculated from the model [22] according to,

$$
\sigma_{e f f}=\sigma\left[1-\left(\frac{f\left(S_{e f f} / 2+1\right)}{f\left(S_{\text {eff }} / 2+1\right)+(1-f)}\right)\right]=\sigma-\sigma\left(\frac{f\left(S_{e f f} / 2+1\right)}{f\left(S_{\text {eff }} / 2+1\right)+(1-f)}\right)=\sigma-\sigma_{T}
$$

where $f$ is the volume fraction of the reinforcement. Equation (5) predicts that the stress borne by the reinforcement is linearly dependent on $\sigma$, with the term in parenthesis the proportionality constant. The equation is valid, as mentioned, for the case of composites with elongated and misaligned particles. It is simplified in the case of composites with equiaxial particles, for which $\theta=0$, and the density function is $\chi(\theta)=1$. Furthermore, assuming that particles are equiaxial cylinders aligned with the loading direction, we have $S_{\text {eff }}=1$ (for the case of spherical particles it would be, $S_{\text {eff }}=1.25$ [15]).

Hereafter, we will refer to $\Delta^{\prime} \sigma(\sigma)$ as the total composite creep strength increment with stress and to $\Delta \sigma(\sigma)$ as the composite creep strength increment after subtracting the matrix strengthening factor, following the same notation as in [1].

\section{4.- Data analysis and discussion}

The PM materials of Table I have been sub-divided in two groups according to the morphology of the reinforcement, namely: materials reinforced by equiaxial particles [5-9] and materials reinforced by elongated particles (whiskers/short fibers) [1,10-15]. This distinction is based on their different creep strength increment, $\Delta^{\prime} \sigma(\sigma)$, with respect the corresponding un-reinforced alloys (see figure $6 \mathrm{a}$ in [1]), and also on the calculation from the model (equation 5). The composites with elongated particles reveal, in general, a rapid and monotonic increase of the strength increment with $\sigma$. On the other hand, in the composites with equiaxial particles the slope of $\Delta^{\prime} \sigma v s . \sigma$ data is lower and more erratic. Both the equiaxial and elongated particle reinforced composites, however, present, at least, a remarkable trend of the strength difference to increase with the applied stress (see figure 6 of [1]). It is worth mentioning the different processing sensitivity of the mechanical properties and model's predictions of these two groups of composites. For the materials with equiaxial particles, the aspect ratio of the reinforcement is about unity, a value which is maintained during material processing (particle breakage barely occurs if particle size is sufficiently small). This indicates that processing parameters are not relevant in establishing the mechanical properties of the composite. In other words, equiaxial particle reinforced composites are moderately processing sensitive. On the other 
hand, the aspect ratio and degree of orientation of the elongated particles with the externally applied stress of these composites are strongly dependent on the processing parameters and also important parameters to determine their mechanical properties. This reveals the high processing sensitivity of the mechanical properties of these composites materials [24].

\section{a) Equiaxial particle reinforced composites}

The comparison of the creep strength increase, $\Delta \sigma$, of these composites (after microstructural strengthening subtraction) with Ryu's et al [22] Shear-Lag model prediction of the load transferred to the reinforcement is summarized in the plot of figure 1a. For better comparison of trends, the data in the low stress range are shown in a magnified plot in figure 1b. As can be seen, a reasonable scatter is appreciated, but the predictions for each composite is in remarkably good agreement with the data of the increased creep strength considering the very simple approach assumed (no damage or de-cohesion at metal-ceramic interface and not other strengthening mechanism is considered). As described in [1], the data increase of $\Delta \sigma v s . \sigma$ of this group of composites [5-9], occurs in a rather erratic manner. Furthermore, the data and slope trends for the different composites spread over a wide range of values. All this can be understood from either of the following two different phenomena or both: a) The different underlying damage processes occurring during creep deformation in these composites. The data scatter may be associated with the influence of irregular damage processes at metal-matrix interfaces. b) A process of load transfer relaxation by diffusional flow can also account for the erratic $\Delta \sigma v s . \sigma$ behavior. As argued in [13], this process is noticeable if particles size is sufficiently small.

It is to be noted that the model's prediction and the experimental data for the composite of reference [8] data is excellent in the low range of applied stress, but deviates quite remarkably at higher stresses. The data in the high stress range correspond to tests conducted at the lowest temperature of testing $(623 \mathrm{~K})$. It is likely that strengthening mechanisms associated with low-temperature behavior, such as the increased dislocation density and the interaction of geometrically necessary dislocations (GNDs), could play a role in composite creep strengthening, explaining the divergence from the model's prediction, as proposed in [1].

\section{b) Elongated particle reinforced composites}

This group of composites shows two separated behaviors, as it is shown in the plot of $\Delta \sigma v s . \sigma$ of Figure 2. Figure $2 a$ ) is for all the data analyzed and figure $2 b$ ) shows the detail for the data in the range of low applied stress. Firstly, there are some composites $[10,13,15]$ which show a remarkably linear dependence of $\Delta \sigma$ with $\sigma$, and the slope (or proportionality) is high and quite similar among them. The effectiveness of load transfer is about $50 \%$, or slightly higher. Furthermore, the model's 
predictions are also in quite good harmony with the experimental data of composites creep strength increment. In the second set of composites $[1,11,12,14]$, the linearity is not so remarkable as for the first group, and the slope is also lower. The behavior of $\Delta \sigma v s . \sigma$ of this group of composites is in fact, very similar to that of the equiaxial particle reinforced composites, Figure 1 and Table I. The $\Delta \sigma(\sigma)$ dependence predicted by the Shear-Lag model is also in good agreement with the experimental values found.

Some important remarks must be done for materials from references [12-14]. The lack of information of the reinforcement aspect ratio has been overcome assuming statistical values derived from detailed investigations in extruded aluminum MMCs [1,11,15]. Furthermore, a study of the dependence of $\Delta \sigma(\sigma)$ predicted by the Shear-Lag model with different values of $S_{\text {eff }}$ for these composites has been carried out to validate the rigor of the model's prediction. In figure 3 the experimental data of $\Delta \sigma(\sigma)$ are represented together with the Shear-Lag model predictions with $S_{\text {eff }}$ values of 6.0 and 9.0 for composite of reference [13] and of 1.5 and 3.0 for composites of references $[12,14]$, as indicated in the figure. As it is seen, the predictive capacity of the Shear-Lag model is good enough to maintain the ideas defended for these composites.

Finally, $t$ is also of particular relevance the composite of reference [13], in which the reinforcement is able to sustain a large stress, allowing the material to maintain an applied stress as high as 400 $\mathrm{MPa}$ at $648 \mathrm{~K}$, figure 4 . This stress is partitioned between the reinforcement and the matrix according to the trend shown in the plot of figure 4: The reinforcement bears some $215 \mathrm{MPa}$ (strength increment at $400 \mathrm{MPa}$ ), and the remaining stress, some $185 \mathrm{MPa}$, is borne by the $8009 \mathrm{Al}$ matrix alloy. This stress is nearly the yield stress of the 8009Al alloy at this temperature [5]. This means that, the aspect ratio of the reinforcing particles in this material (7.4) is so high, than it can bear completely the fraction of load transferred from the matrix. In fact, the average stress that the individual fibers bear, as calculated by the rule of mixtures [1], goes up to almost $1.5 \mathrm{GPa}$. Therefore, the limiting factor for the load partitioning process is the yield stress of the matrix. This explanation to understand the improved creep strength of this composite differs from that proposed in [13]. In that work, the authors propose that the improved composite behavior is attributed to the magnification of a threshold stress term by a load transfer factor. As seen, this view differs from the simpler explanation proposed in the present research. High values of the load transferred to the reinforcement have been obtained in similar composite from in situ direct measurements by neutron diffraction [25]. In this case, a value of $0.25 \mathrm{GPa}$ has been measured (see Table I of [25]). This value is, in principle, relatively low in comparison to that calculated for the composite of ref. [13]. This is because pure $\mathrm{Al}$ is used as the metallic matrix in the composite studied, which has a much lower yield stress than the 8009Al alloy used in [13]. Furthermore, it also implies that the maximum 
load that the composite can sustain is low. Additionally, the aspect ratio of the reinforcement in the composite studied in [25] is also lower than in [13].

From the differences found between the behaviors of these two groups of PM composites some reasonable and consistent implications can be derived in regard the bonding of the metal-ceramic interface which has occurred during materials processing. With elongated particles the total metalceramic surface where shear deformation occurs to achieve the bonding at the metal-ceramic interface is higher than in the equiaxial particle composites. Typically, these elongated particles tend to align with the extrusion axis direction during composite consolidation at elevated temperatures. Plastic flow in solid state occurring near the interfaces is responsible of the "firm" bonding when shear deformation predominates. This circumstance is important in the composites with elongated particles, but not so relevant when the particles are equiaxial. For these cases, the occurrence of some de-cohesion or damage mechanisms are, hence, more likely to occur than in elongated particle reinforced composites, in agreement with the trends noted.

\section{5.- Summary}

A quantitative and thorough study of the creep strengthening of a variety of PM composites reported from the literature is presented. These composites are more appropriate than IM ones to go deep in understanding their increased creep strength. This is because they develop a stronger bonding during materials processing than IM composites and, hence, damage or de-cohesion mechanisms at the interface are less likely to occur during composite deformation. The experimental creep strength increment of these composites has been evaluated provided that creep data of the corresponding unreinforced alloys is also available. The increased creep strength, after subtracting the factor associated to the microstructure, has been compared successfully with the load transferred to the reinforcement predicted by a simple Shear-Lag model. The new comparisons presented between experimental data and model's predictions, particularly for the composite reinforced by elongated particles, sustain more thoroughly the relevance of the load transfer mechanism during creep of MMCs defended in a previous work.

\section{Acknowledgements}

Financial support from project MAT05-00527, MEC, Spain, is acknowledged. 


\section{References}

1.- Fernández R, González-Doncel G. Acta Mater. 2008;56:2549-62.

2.- Spigarelli S, Cabibbo M, Evangelista E, Langdon TG. Mater Sci Eng A 2002;328:39-47.

3.- Huang M, Li X, Yi H, Ma N, Wang HJ. J Alloys Compd 2005;389:275-80.

4.- Moreno MF, González Oliver CJR. Mater Sic Eng A 2006;418:172-81.

5.- Zedalis MS, Bryant JD, Gilman PS, Das SK. JOM 1991;8:29-31.

6.- Li Y, Langdon TG. Metall Mater Trans A 1998;29:2523-31.

7.- Desmukh SP, Mishra RS, Keding KL. Mater Sic Eng A 2005;410-411:53-7.

8.- Čadek J, Kuchařová K, Zhu SJ. Mater Sci Eng A 2000;283:172-80.

9.- Park K-T, Lavernia EJ, Mohamed FA. Acta Metall Mater 1990;38:2149-59.

10.- Pickens JR, Langan TJ, England RO, Liebson M. Metall Trans A 1987;18:303-12.

11.- N.Hansen, D.J.Jensen, Y.-L. Liu. Proc. 9th Int. Riso Symp. 1988. p.365-70.

12.- Zhu SJ, Peng LM, Ma ZY, Bi J, Wang FG, Wang ZG. Mater Sic Eng A 1996;215:120-4.

13.- Kuchařová K, Čadek J, Zhu SJ. J Mater Sci 2003;38:3535-43.

14.- Peng LM, Zhu SJ, Ma ZY, Bi J, Wang FG, Chen HR, Northwood DO. Mater Sci Eng A 1999;265:63-70.

15.- Ryu HJ, Chung H, Seung IC, Hong SH. J Mater Res 2004;19:3633-40.

16.- Lloyd DJ. Comp Sci Technol 1989;35:159-79.

17.- Frost HJ, Ashby MF, in Deformation Mechanisms Maps, Pergamon Press, Oxford, 1982.

18.- Fernández R, González-Doncel G. J Alloys Compd 2007; 440:158-67.

19.- Estrin Y. Mater Sci Eng A 2007; 463:171-6.

20.- Cox HL. British J. Appl Phys 1952;3:72-8.

21.- Eshelby JD. Proc Roy Soc A 1957; 241:376-96.

22.- Ryu HJ, Cha SI, Hong SH. J Mater Res 2003;18:2851-8.

23.- Nardone VC, Prewo KM. Scripta Metall 1986;20:46-8.

24.- Borrego A, Fernández R, Cristina MC, Ibáñez J, González-Doncel G. Comp Sci Technol 2002;62:731-42.

25.- Winand HMA, Whitehouse AF, Withers PJ. Mater Sci Eng A 2000;284:103-13. 


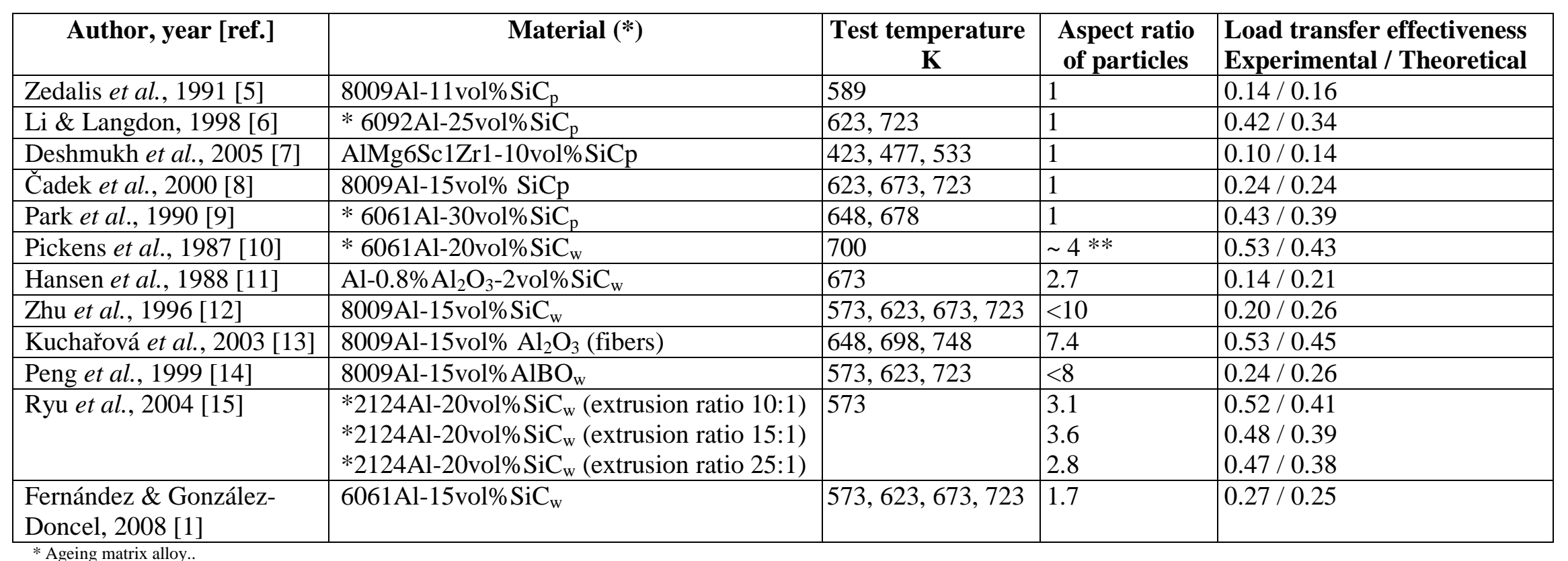

** Approximate value estimated from micrograph of figure 5 of ref. [10]

Table I. Summary of the creep studies on discontinuously reinforced PM MMCs (aluminum alloy matrix) which include data of the corresponding un-reinforced alloys. 


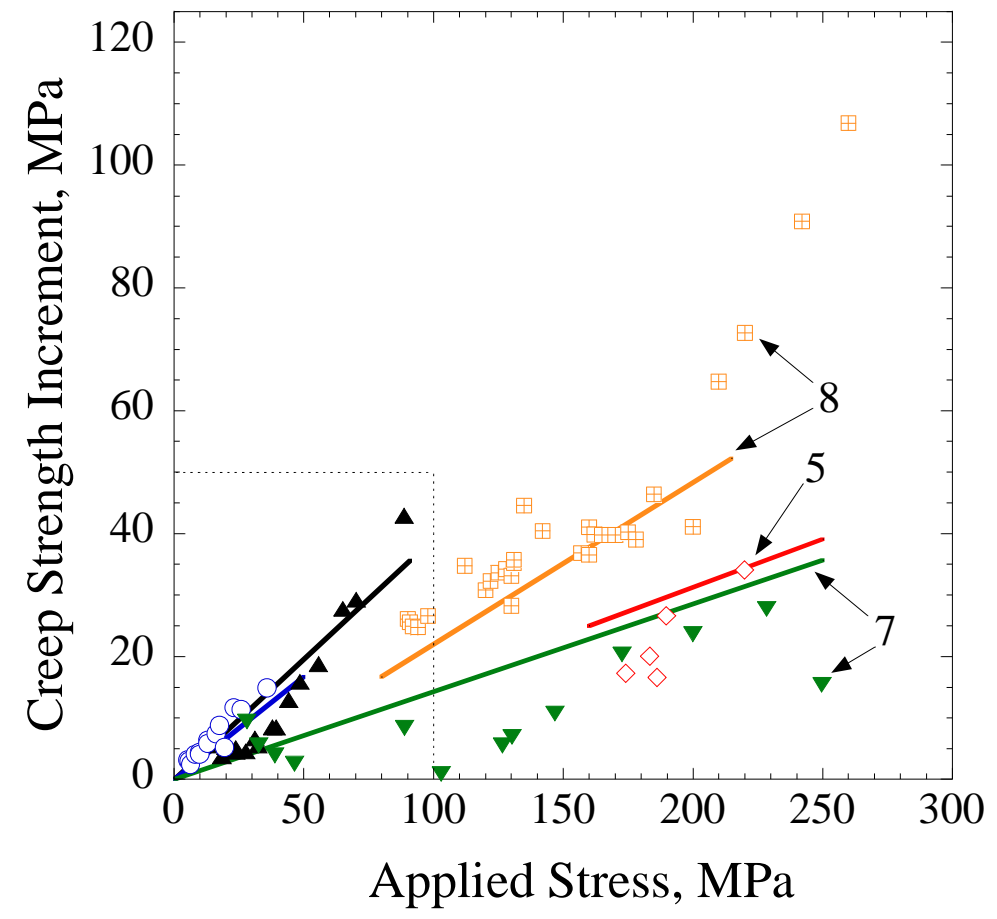

a)

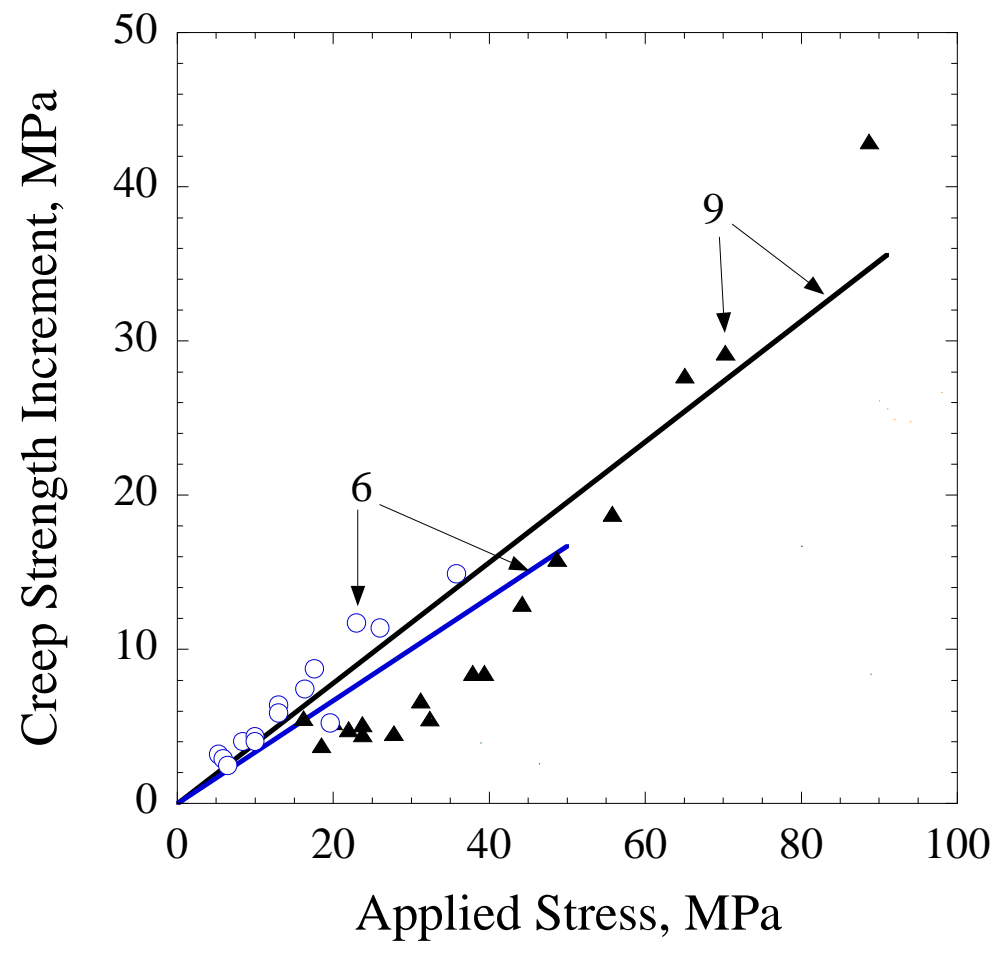

Figure 1. a) Creep strength increment as a function of the applied stress and Ryu's et al Shear-Lag model's prediction (solid lines) for the equaxial particle reinforced composites. b) Detail of data and model prediction in the low stress range. Numbers denote the corresponding reference number. 


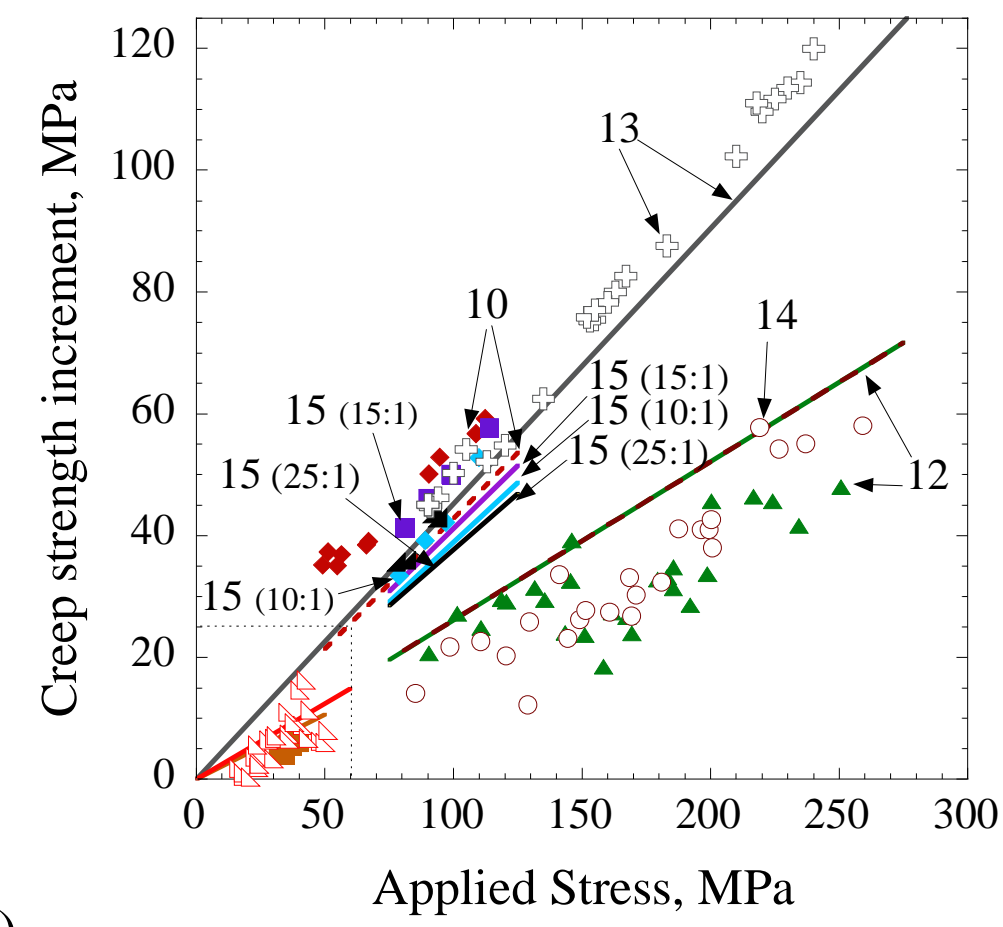

a)

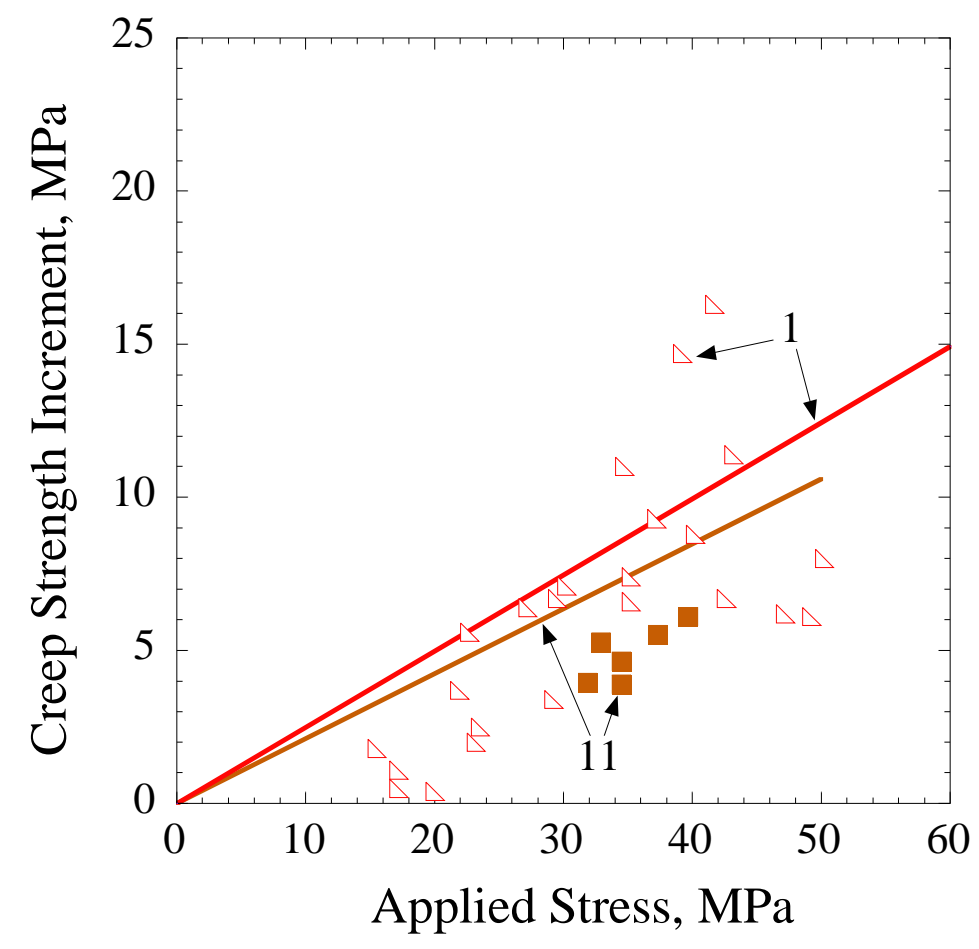

b)

Figure 2. a) Creep strength increment as a function of the applied stress and Ryu's et al Shear-Lag model's prediction (solid lines) for the elongated particle reinforced composites. b) Detail of data and model prediction in the low stress range. Numbers denote the corresponding reference number. Numbers in parenthesis of materials of reference [15] denote extrusion ratio (seeTable I). 


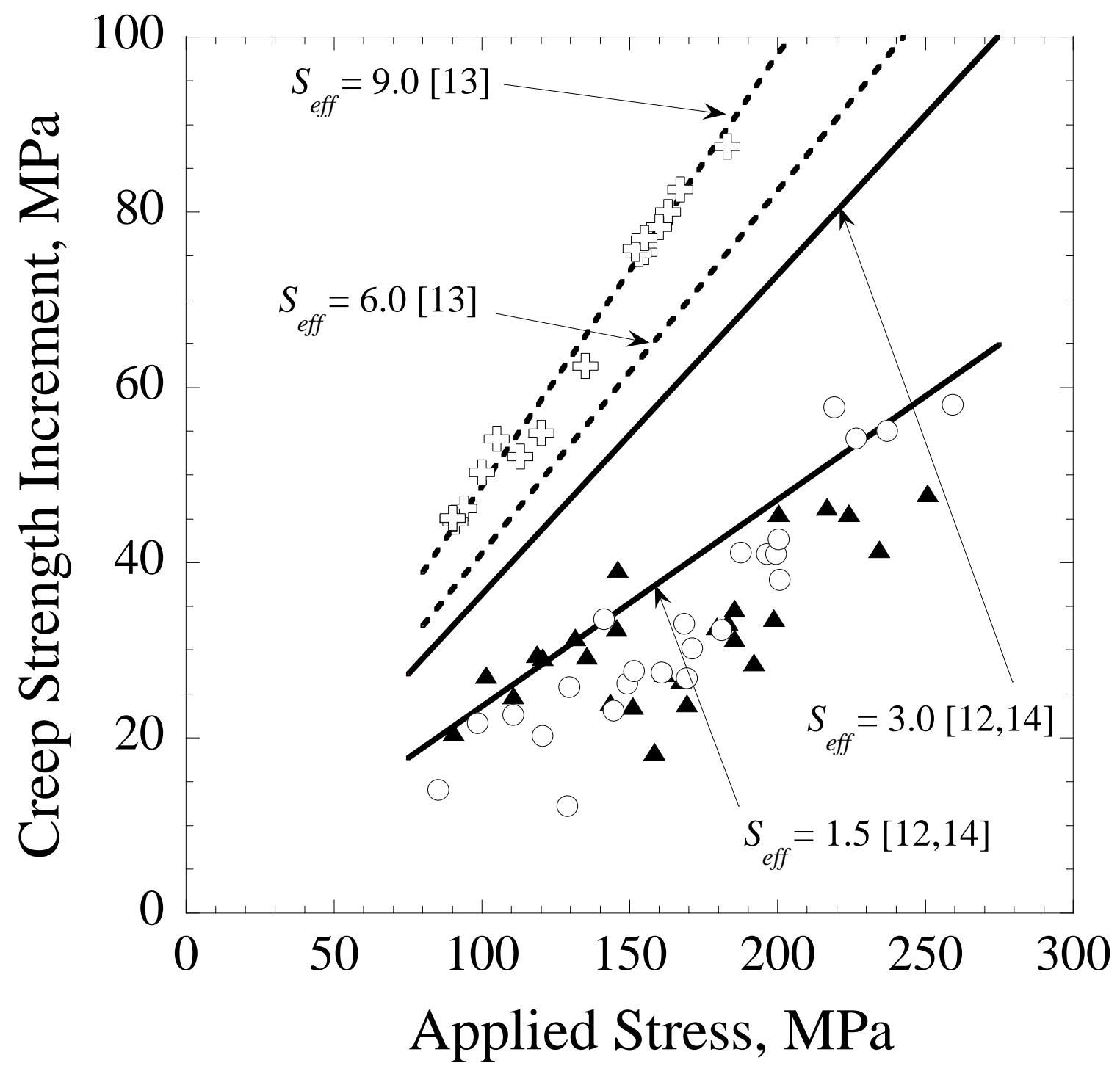

Figure 3. Dependence of $\Delta \sigma(\sigma)$ by the Shear-Lag model prediction of the creep strength increment for values of $S_{\text {eff }}$ and comparison with the experimental data for the materials investigated in references [12-14]. 


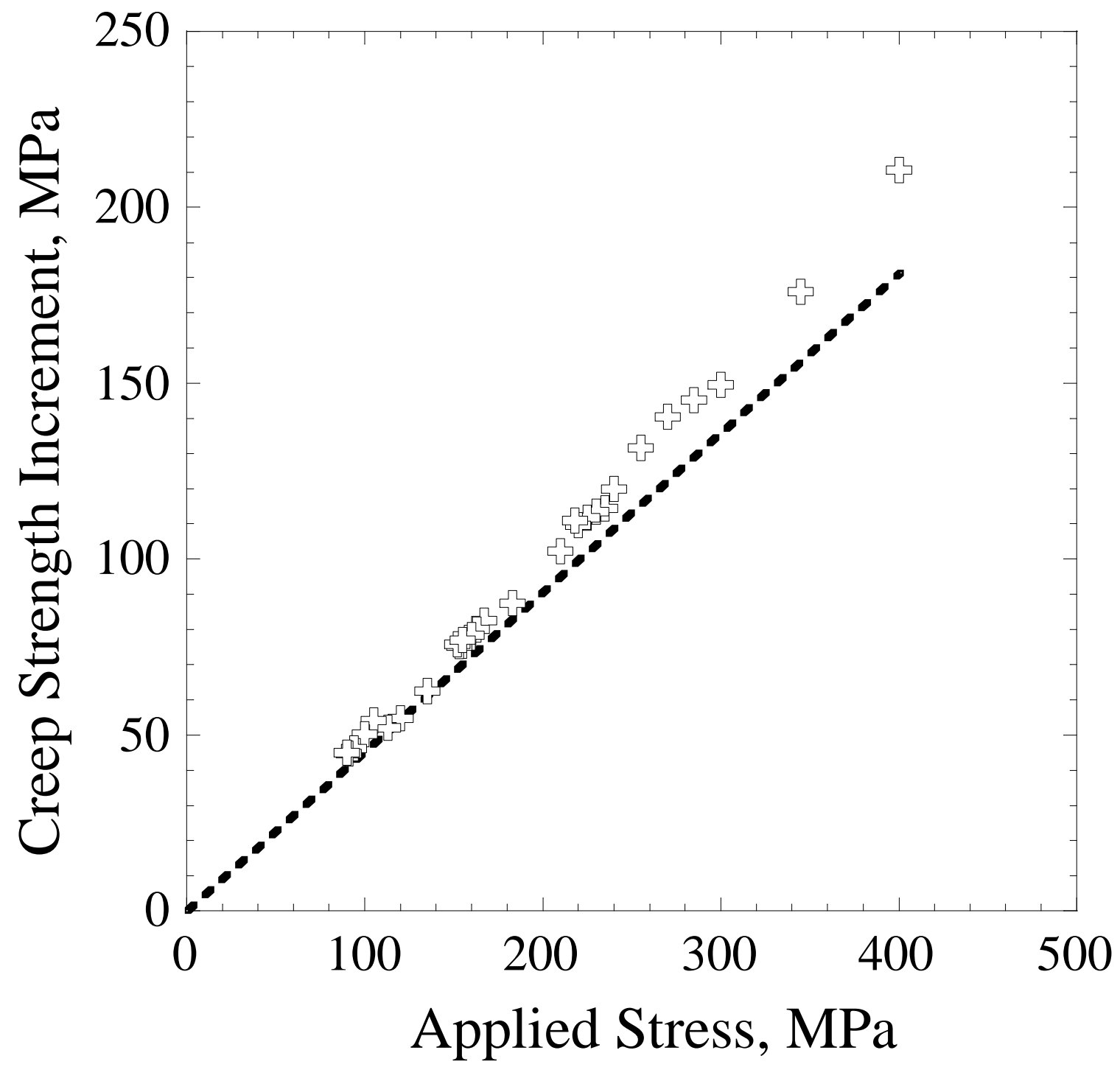

Figure 4. Creep strength increment data as a function of the applied stress and Shear-Lag model's prediction of the load transferred to the reinforcement for composite of reference [13]. Model's prediction assumes an aspect ratio of the reinforcement of 7.4. 


\section{List of figures}

Figure 1. a) Creep strength increment as a function of the applied stress and Ryu's et al Shear-Lag model's prediction (solid lines) for the equaxial particle reinforced composites. b) Detail of data and model prediction in the low stress range. Numbers denote the corresponding reference number.

Figure 2. a) Creep strength increment as a function of the applied stress and Ryu's et al Shear-Lag model's prediction (solid lines) for the elongated particle reinforced composites. b) Detail of data and model prediction in the low stress range. Numbers denote the corresponding reference number. Numbers in parenthesis of materials of reference [15] denote extrusion ratio (seeTable I).

Figure 3. Dependence of $\Delta \sigma(\sigma)$ by the Shear-Lag model prediction of the creep strength increment for values of $S_{\text {eff }}$ and comparison with the experimental data for the materials investigated in references [12-14].

Figure 4. Creep strength increment data as a function of the applied stress and Shear-Lag model's prediction of the load transferred to the reinforcement for composite of reference [13]. Model's prediction assumes an aspect ratio of the reinforcement of 7.4. 